\title{
SCORPIO-2 Guiding and Calibration System in the Prime Focus of the 6-m Telescope
}

\author{
V. L. Afanasiev, ${ }^{1, *}$ V. R. Amirkhanyan, ${ }^{1,2}$ A. V. Moiseev, ${ }^{1}$ R. I. Uklein, ${ }^{1}$ and A. E. Perepelitsyn ${ }^{1}$ \\ ${ }^{1}$ Special Astrophysical Observatory, Russian Academy of Sciences, Nizhnii Arkhyz, 369167 Russia \\ ${ }^{2}$ Sternberg Astronomical Institute, Lomonosov Moscow State University, Moscow, 119991 Russia
}

(Received July 10, 2017; Revised September 14, 2017)

\begin{abstract}
We describe a device (adapter) for off-axis guiding and photometric calibration of wideangle spectrographs operating in the prime focus of the 6-m telescope of the Special Astrophysical Observatory of the Russian Academy of Sciences. To compensate coma in off-axis star images an achromatic lens corrector is used, which ensures maintaining image quality $(F W H M)$ at a level of about $1^{\prime \prime}$ within $15^{\prime}$ from the optical axis. The device has two $54^{\prime \prime}-$ diameter movable guiding fields, which can move in $10^{\prime} \times 4.5$ rectangular areas. The device can perform automatic search for guiding stars, use them to control the variations of atmospheric transmittance, and focus the telescope during exposure. The limiting magnitude of potential guiding stars is $m_{R} \sim 17^{\mathrm{m}}$. The calibration path whose optical arrangement meets the telecentrism condition allows the spectrograph to be illuminated both by a source of line spectrum (a He-Ne-Ar filled lamp) and by a source of continuum spectrum. The latter is usually represented either by a halogen lamp or a set of light-emitting diodes, which provide illumination of approximately uniform intensity over the wavelength interval from 350 to $900 \mathrm{~nm}$. The adapter is used for observations with SCORPIO-2 multimode focal reducer.
\end{abstract}

\section{INTRODUCTION}

The six-meter Big Telescope Alt-Azimuth (BTA) is the first large telescope on an altazimuth mounting with machine-controlled tracking of target objects [1]. This was a pioneering technical solution for up to the time (late 1960s) which gave rise to the use of altazimuth mounts for large, massive telescopes. Besides the specifics of guiding, which required the use of a computer-assisted control system, altazimuth mounting is, unlike parallactic mounting, distinguished by the rotation of the field of view. Note that the accuracy of the tracking of the observing object is determined not only by the quality of telescope mechanics, but also by the variation of atmospheric conditions.

Electronic address: vafan@sao.ru 
Therefore long exposures in a large field of view require off-axis guiding to be performed using at least two guide stars. Whereas one star is used to correct the guiding of the telescope mounting, the second star serves to take into account the error of the compensator of the rotation of the field of view. The latter is maximal in observations made in the prime vertical and at small zenith angles, where the rate of change the parallactic angle is the fastest. The telescope was initially equipped with a standard Ricci cassette meant for photographic observations in the prime focus within a 18'-diameter field. This device had two movable off-axis guiding microscopes.

Mounting of heavy-weight electrooptical devices (long-slit, multiobject, and integral-field spectrographs, focal reducers, etc.) in the prime focus of the 6-m telescope for recording images in a large field of view made it necessary to seek new design solutions for off-axis guiding. The use of schemes like the Cassegrain adapter of the 3.6-m ESO telescope [2] in the prime focus is fraught with a number of problems. The high focal ratio $(f / 4)$ and small offset of the focal plane above the plane of the rotating table make it impossible to implement the optomechanical scheme with movable fields using lens collectives.

An adapter was developed for the prime focus of the 6-m telescope. It underwent a stage-bystage upgrade, which included incorporating results of new technologies and software solutions. As of now, there have been four versions of this adapter:

1. In the first version of the adapter guiding field images were transferred to a single field via optical fiber bundles. The entrance ends of the bundles could move in the radial and tangential directions in the focal plane of the telescope, and the exit ends were fixed in the focal plane of the guiding eyepiece. Stars were searched manually by the observer, and guiding by stars was performed visually. On the 6-m telescope such guiding scheme was first used in 1979 for the optical identification of 5C survey radio sources using McMullan electronographic camera [3]. The good guiding quality made it possible to acquire very deep images with this camera and observe faint galaxies down to 25th magnitude [4].

2. The next version of the adapter was equipped with a wavelength calibration lamp, and the images from fiber bundles were transferred to LI-702 TV camera and preamplified with an image converter tube. The adapter was controlled by a remote controller and a long communication link from the observers' room located inside the dome of the 6-m telescope. Beginning with 1990 this adapter was used for observations with three versions of MPFS integral-field spectrograph [5, 6] and MOFS multiobject spectrograph [7]. It was also used for the first observations with SCORPIO multimode prime focus reducer [8]. 
3. Beginning with 2001, observations with SCORPIO and MPFS instruments continued with a new adapter having fundamentally upgraded design: lens correctors of guiding fields were mounted to correct the coma of the primary mirror, a "flatfield" lamp, a bundle focusing mechanism, and an adjustable crosshair illumination were added, and calibration optics were upgraded to ensure telecentric illumination from the integrating sphere. The movable units and lamps were controlled by a microprocessor, which, in turn, was controlled by the host computer located at the prime focus of the telescope. Since 2007 the TV tube in the guide view was replaced by a CCD camera. Automatic search for guide stars was implemented based on USNO 2.0 catalog. This adapter version, which was described in [8,9], is still used (summer of 2017) with SCORPIO instrument for remote observations performed from the Main Laboratory building of the SAO RAS, $5 \mathrm{~km}$ from the $6-\mathrm{m}$ telescope.

In this paper we present the new, fourth version of the adapter. It was designed based on our wide experience from previous modifications, and incorporating such capabilities as correction of the telescope focus during exposures and control of atmospheric transmittance. Section 2 describes the overall layout of the adapter and particularities of its implementation; Section 3 describes guiding and the properties of the view, Section 4 discusses the features of the calibrating unit, and the Conclusions section presents the main points of the work.

\section{DESCRIPTION OF THE ADAPTER}

\subsection{General Scheme}

The adapter is mounted on a rotating table in the prime focus of the $6-\mathrm{m}$ telescope and is used both for offset guiding and for telecentric illumination of the entrance pupil of the instrument installed on it by various light sources. The adapter can be used for mounting SCORPIO-2 focal reducer and other spectrographs with mass no greater than $150 \mathrm{~kg}$ and flange focal distance no greater than $40 \mathrm{~mm}$.

Figure 1 shows the optical layout of the adapter. The adapter has two movable fields of view to search for guide (reference) stars. The light from such a star first comes to off-axis lens corrector (3), which compensates coma of the primary mirror of the telescope. Then, diagonal mirror (4)redirects the light beam to the entrance end of fiber bundle (5), which together with the mirror is moved across the field in two perpendicular directions by STANDA linear translation stages ${ }^{1}$. The fiber

\footnotetext{
${ }^{1}$ http: //www.standa.lt/
} 


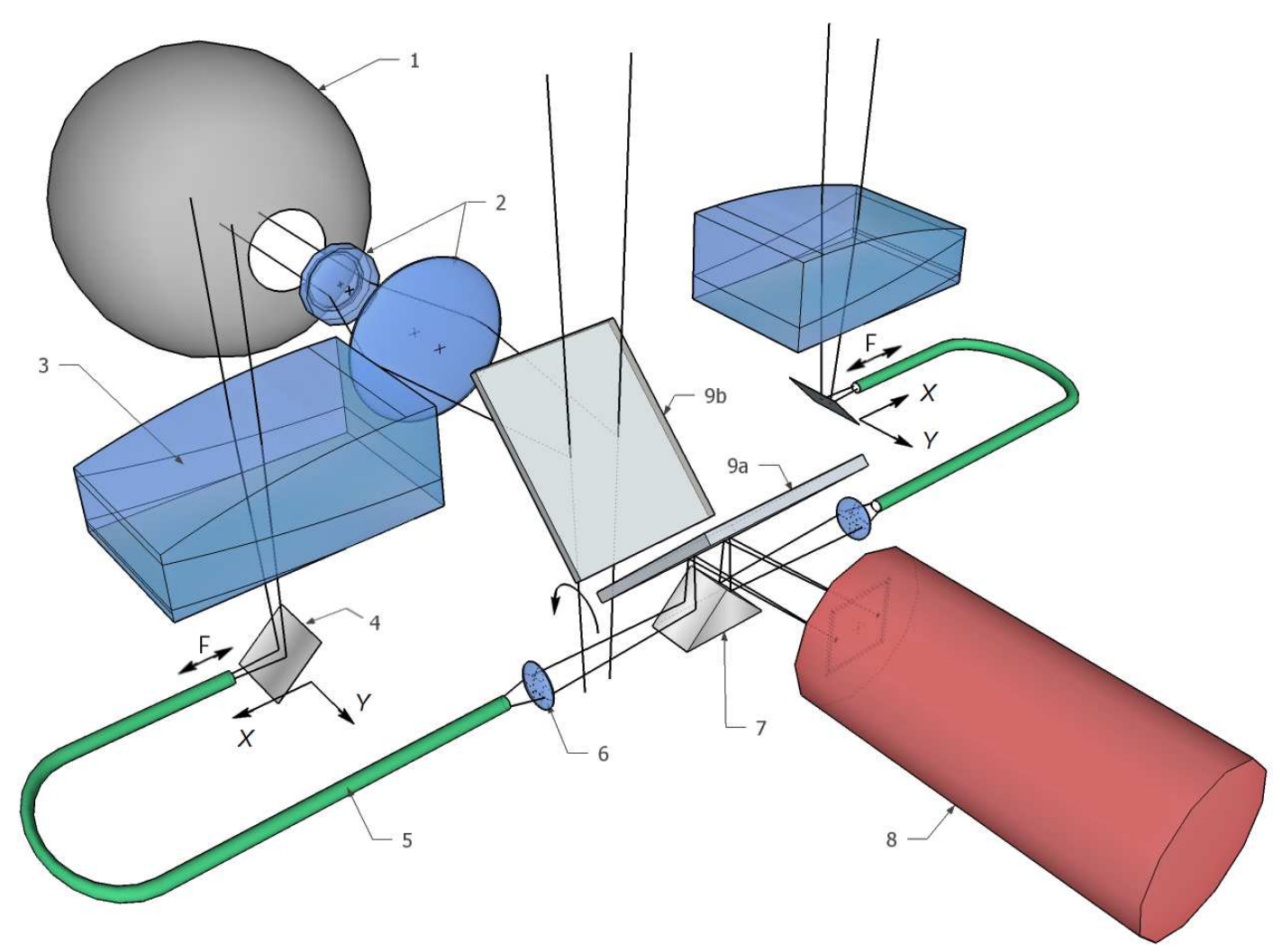

Figure 1. Optical layout of the adapter: (1) - integrating sphere, (2) - calibration illuminator optics, (3) - off-axis lens corrector, (4) - mirror, (5) - fiber bundle, (6) - fiber lens, (7) - reflecting prism, (8) guiding CCD, (9) - diagonal mirror (both positions are shown: (9a) - FIBERS, (9b) - FIELD).

bundle has a diameter of $54^{\prime \prime}$ in sky projection, and can move within a $10^{\prime} \times 44^{\prime \prime} 5$ field. The centers of guide fields are located at a distance of $12^{\prime}$ from the center of the fields of view of the system. The relative arrangement of the fields is presented in Subsection 3.2. The images of the exit ends of the bundles are projected by lens (6) and directed by prism (7) and diagonal mirror (9) onto the focal plane of guiding CCD (8). Mirror (9) has two working positions - FIBERS (9a) and FIELD (9b). Figure 1 shows both positions. In the FIBERS position light from observed objects is received by the equipment mounted on the adapter (the mirror is offset from the beam), and the guiding CCD captures images of guide stars. In the FIELD position the diagonal mirror transfers the image of the science field of view to the guide view (the mirror covers the central beam), making it possible to perform field identification and to position bright objects onto the slit. The size of the FIELD field on the view is $3^{\prime} \times 2^{\prime}$. The same mirror position is used to calibrate the main CCD using integrating sphere (1). For a more detailed description of the calibrating unit see Section 4. 


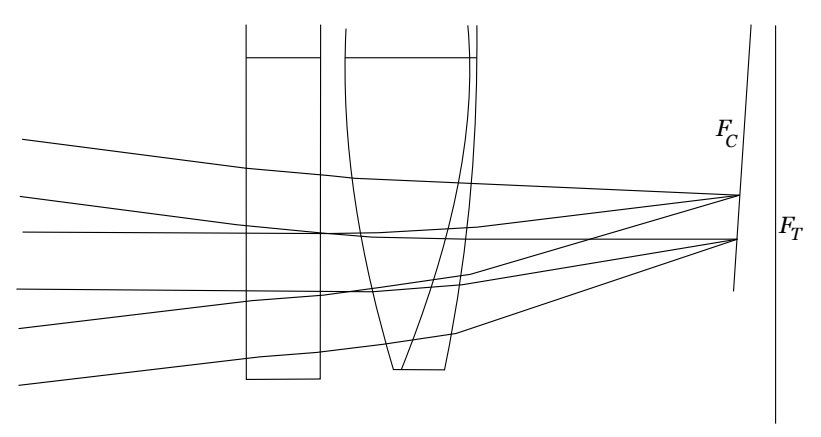

Figure 2. Optical layout of the off-axis lens corrector.

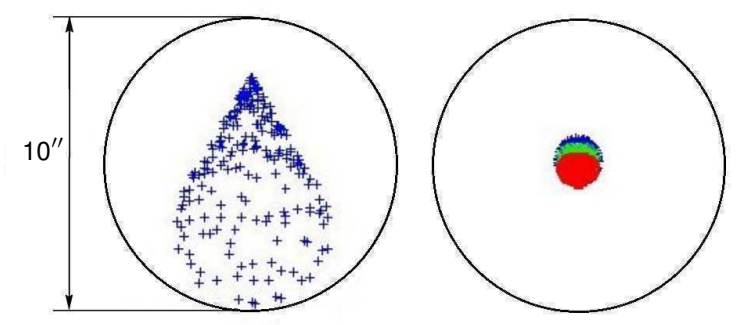

Figure 3. Computed point diagrams at about $12^{\prime}$ offset from the axis of the primary mirror: without (left) and with (right) the corrector.

\subsection{Off-axis Lens Corrector}

The main problem in offset guiding on the 6-m telescope is coma aberration of the parabolic mirror. Standard prime focus corrector compensates aberrations in the $18^{\prime}$ large field and cannot be used in our scheme, because the instruments mounted onto the adapter work without lens corrector. We developed and constructed a relatively simple two-lens corrector made of domestic-manufactured K8 and TFZ glass. The corrector is achromatized in the $0.5-0.7 \mu \mathrm{m}$ wavelength interval and yields an equivalent telescope focal distance of $18.5 \mathrm{~m}$. Figure 2 shows the optical layout of the corrector. The focal surface of the corrector $F_{C}$ within the range of motion of the fiber bundle is flat to the first approximation. It is tilted by 3.9 with respect to the telescope focal plane $F_{T}$.

Figure 3 shows the result of coma correction at an offset of about $12^{\prime}$ from the primary mirror axis. Figure 4 shows the computed polychromatic point-spread function of the corrected image. As is evident from the figure, the seeing $(F W H M)$ of the image produced with the use of the corrector is $40 \mu \mathrm{m}$ or better, which corresponds to about $1^{\prime \prime}$; it is quite sufficient for guiding purposes. The optical layout was computed using ZEMAX program ${ }^{2}$.

\footnotetext{
${ }^{2}$ http://www. zemax.com/
} 


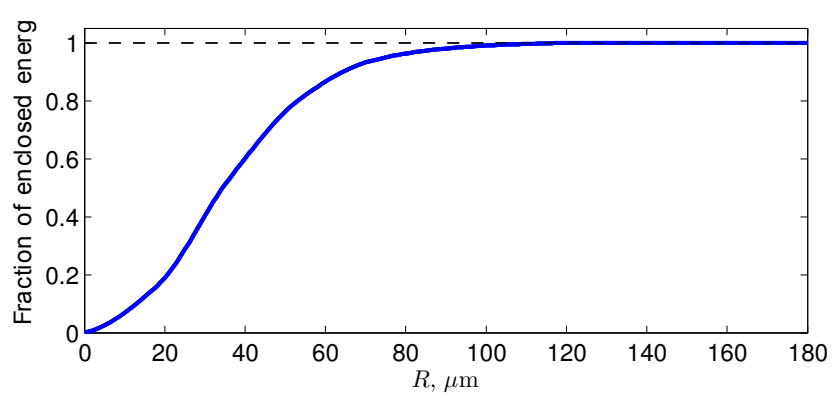

Figure 4. Mean energy concentration in the scattering circle (spot) in the $8-15^{\prime}$ interval of offsets from the optical axis.
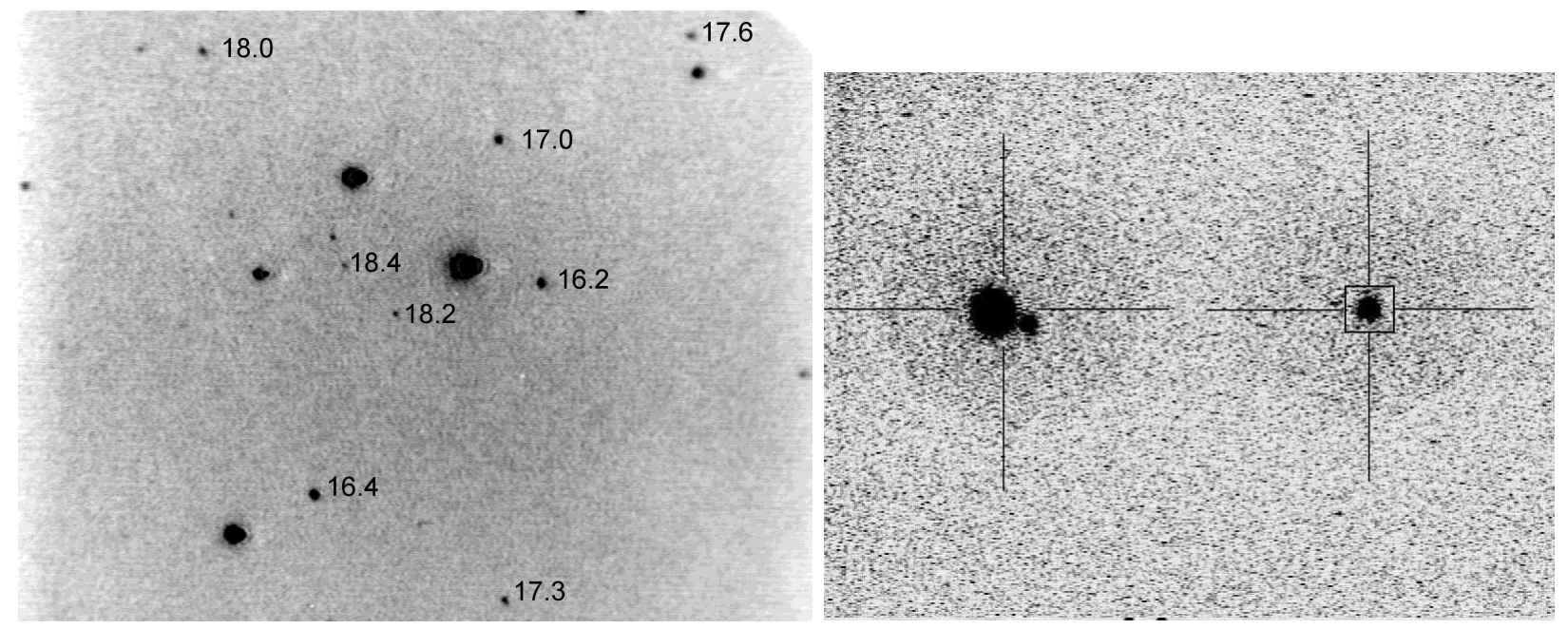

Figure 5. Example of images acquired in the FIELD (left) and FIBERS (right) modes with USNO 2.0 $R$-band magnitudes of faint stars shown.

\subsection{Guide View}

We first used LI-702 TV transmitting tubes with a preamplifier based on an image converter tube with a microchannel plate as guide views. Since 2007 standard industrial 1/2-inch monochrome Sony ICX 429ALL CCD sensor with $582 \times 752$ pixels is used as the guide view. Its parameters are controlled by RS-232 interface, and the image is digitized by a TV tuner. By the end of 2017 it is planned to be replaced by commercial Atik Titan ${ }^{3}$ camera based on a similar Sony ICX424 sensor with the images transmitted to the computer via USB interface.

Figure 5 (left) shows an example of an image of the main science field-FIELD. For clarity of illustration we also show the $R$-band magnitudes of some stars. In the FIELD mode the size of the field of view is $3^{\prime} \times 2^{\prime}$. The right panel in Fig. 5 shows an example of an image from fibers

\footnotetext{
${ }^{3}$ https://www.atik-cameras.com/product/atik-titan/
} 
(FIBERS mode). Because of absorption in fibers, transmission in the FIBERS is twice lower than in the FIELD mode. However, the sensitivity of the guiding CCD is sufficient for reliable detection of moonless sky background.

\subsection{Control System}

The adapter is controlled like other SCORPIO-2 units. Two boards have been developed that are equipped with ATmega8535L microprocessors and power chips to control seven step motors and adapter calibrations. One board controls four motors used to move two guide fields along the $X$ and $Y$ axes and two motors for focusing these fields. Another board controls the FIELD/FIBERS diagonal mirror switch, line spectrum lamp (NEON), continuum spectrum lamp (QUARTZ), and 32 LEDs that produce the flatfield.

The ranges of all movements are controlled by limit switches. The current position $(X, Y)$ of guide fields and the focus are computed from the number of steps made from the initial position. External control commands are transferred to the microprocessor via standard RS-422 interface from the industrial computer operating under Windows OS. Microprocessor programs are set for autonomous operation: after receiving a command they run it and report the result when requested by the control computer. The adapter control interface is written in IDL language ${ }^{4}$ and is incorporated into the overall SCORPIO-2 control software.

\section{GUIDING}

After pointing the telescope to the target object and before starting the guiding the observer should perform a number of preliminary procedures including a search for guide stars, selection of optimum brightness on the view, and preliminary centering. Then the guiding application is run and if the position of the star image centroid differs from the position set by the observer in the guide view the program sends the appropriate correction commands to the telescope control program.

\footnotetext{
${ }^{4}$ http://www.harrisgeospatial.com/
} 


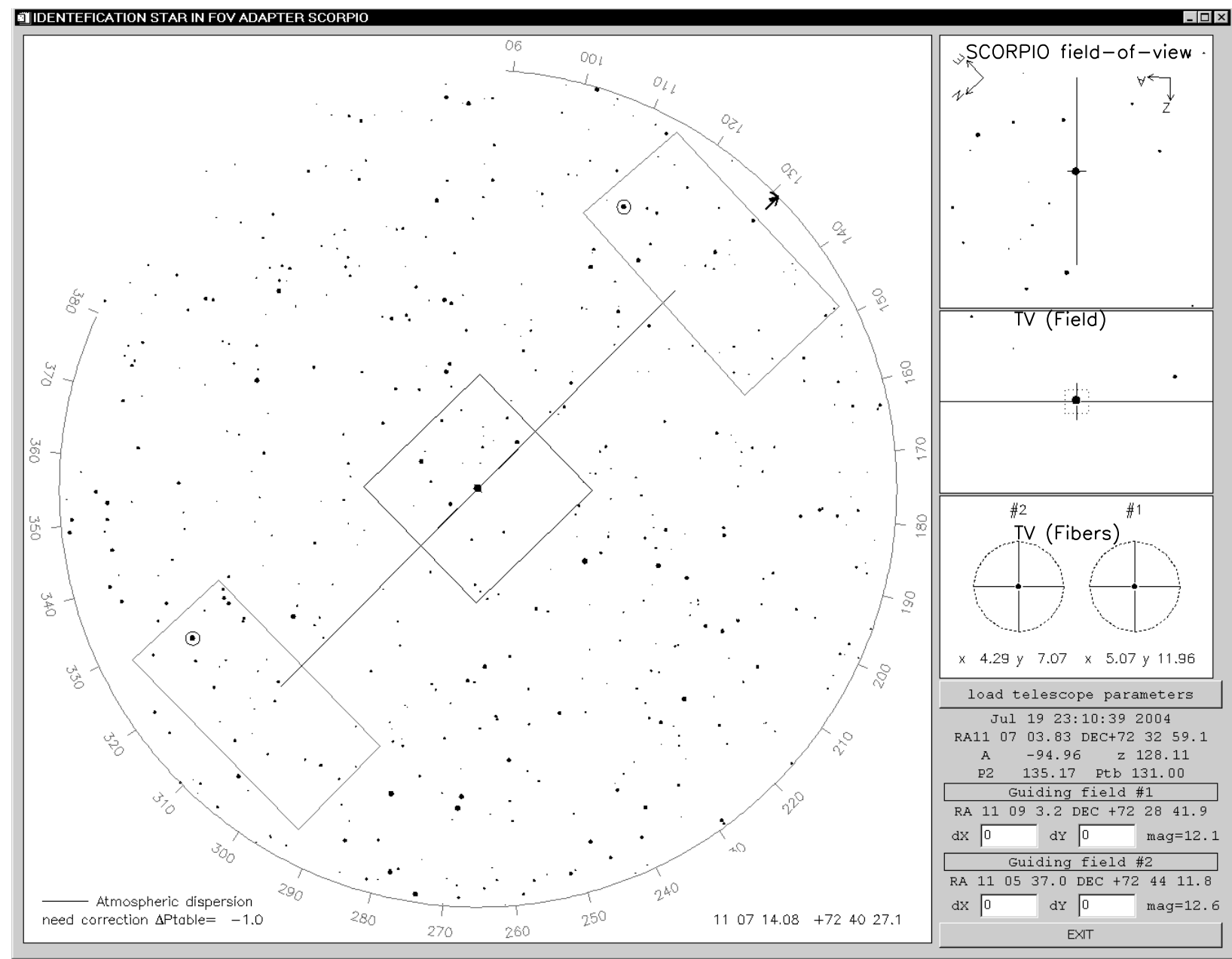

Figure 6. Program interface for searching for guide stars.

\subsection{Program for Searching for Guide Stars}

To accelerate pointing and the search for guide stars, we use IDENTSTAR program written in IDL, which allows computing the current instrumental coordinates of guide stars in the adapter field of view based on the coordinates and orientation of the rotating table transmitted from the control server of the 6-m telescope. Figure 6 shows the appearance of the program interface for searching for guide stars.

When started, the program reads the current telescope coordinates from the server of the 6-m telescope and selects from USNO 2.0 catalog the coordinates of all stars brighter than $20^{\mathrm{m}}$ located in the given sky area within the radius of $15^{\prime}$. The large central square in the window marks the position of the SCORPIO-2 field of view $\left(6^{\prime} \times 6^{\prime}\right)$, and the rectangles indicate the limits of the motion of guide fields inside which the program searches stars for guiding. The small circles whose sizes correspond to the diameters of fiber bundles, indicate the brightest stars, which are automatically 


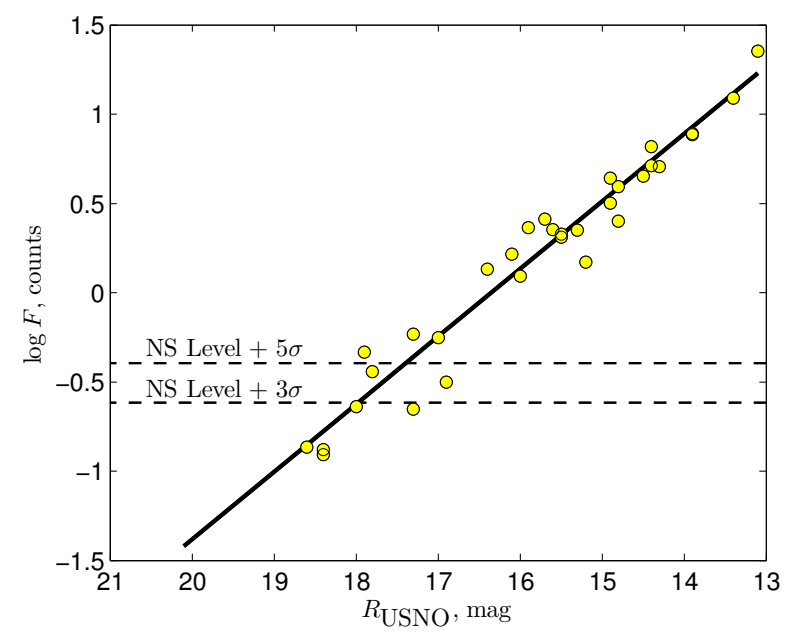

Figure 7. Flux logarithms $\log F$ of guide stars vs. their $R_{\text {USNO-band magnitudes. Images were captured in }}$ the 10-frame moving average mode.

selected by the program. Their magnitudes and positions in the coordinate system of the adapter are indicated in the bottom right fields of the program window. If necessary, the observer can select another guide star among those available in the field. The computed star configuration in terms of FIELD/FIBERS positions is displayed together with the star chart from the catalog. Observational experience shows that it usually takes no more than several minutes to search for and set the guide stars. Note that preliminary setting of stars can be performed while the telescope is repositioned from one object to another.

\subsection{Guiding Program}

For automatic guiding based on a selected star we use our TVGuide program written in IDL, whereas the $\mathrm{C}++$ program written by $\mathrm{E}$. A. Ivanov for operating AverMedia type TV tuners is used to digitize the view image at a $25 \mathrm{~Hz}$ frequency. In addition to visualising the guide view image the program can also superimpose electronic crosshairs and labels. Depending on the adapter mirror position in the large window, the program also displays either the slit position in the FIELD field with the target object or two crosshairs used to aim stars in the FIBERS guiding fields.

\section{3. $\quad$ Limiting Magnitudes}

In the guiding process the tuner polls the guide view output at a frequency of 25 frames per second; to improve the noise properties the images are moving averaged with a 2 to 50 frame window. 

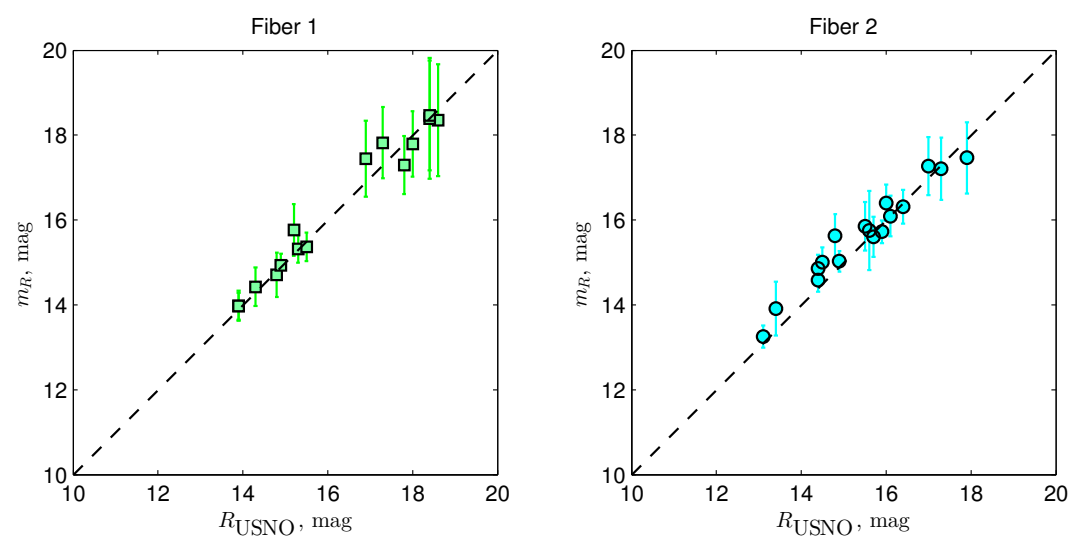

Figure 8. Calibration of the guide view: instrumental magnitudes $m_{R}$ (ordinates) determined with the computed zero point plotted against USNO 2.0 $R$-band magnitudes (abscissae).

The limiting magnitudes of stars suitable for guiding were measured in November 2015 under clear sky and $\theta=1^{\prime \prime}-1^{\prime \prime} .5$ seeing conditions. Figure 7 plots the flux estimates for guide stars against their USNO 2.0 magnitudes. Intensities were precorrected to take into account the gain of the CCD view, which is set separately for each guide star. The dashed lines show the night-sky noise levels for the $3 \sigma_{\mathrm{NS}}$ and $5 \sigma_{\mathrm{NS}}$ detection thresholds. The regression line crosses the dashed lines at $18 \mathrm{~m} 0$ and $17 . \mathrm{m}$, yielding the $R$-band limiting magnitudes for the signal-to-noise ratios of $S / N=3$ and 5 , respectively. The optimum magnitude range for guiding is $10^{\mathrm{m}}-15^{\mathrm{m}}$. Guiding by stars fainter than $15^{\mathrm{m}}$ is possible only under conditions of good seeing and sufficient transmittance.

Note that because of the peculiarities of the control system of the 6-m telescope automatic coordinate correction is performed with a frequency no higher than $0.1 \mathrm{~Hz}$.

\subsection{Control of Atmospheric Transmittance and Guiding Quality}

The zero point for each fiber bundle of the guide view was calibrated using observations of real stars under good transmittance conditions. The results of this calibration are shown in Fig. 8.

After the calibration procedures were performed it became possible to control atmospheric transmittance using guide stars. Note that the calibration relations are further refined during observations. According to our estimates, systematic errors of atmospheric absorption measurements due to the difference between the response curves of the view detector and the $R_{\mathrm{USNO}}$-band filter do not exceed 0.5 .

To control the guiding quality, the user may display the plot showing the current absorption, the size of the image of the guide star and the azimuth and zenith distance offsets (see Fig. 9). 


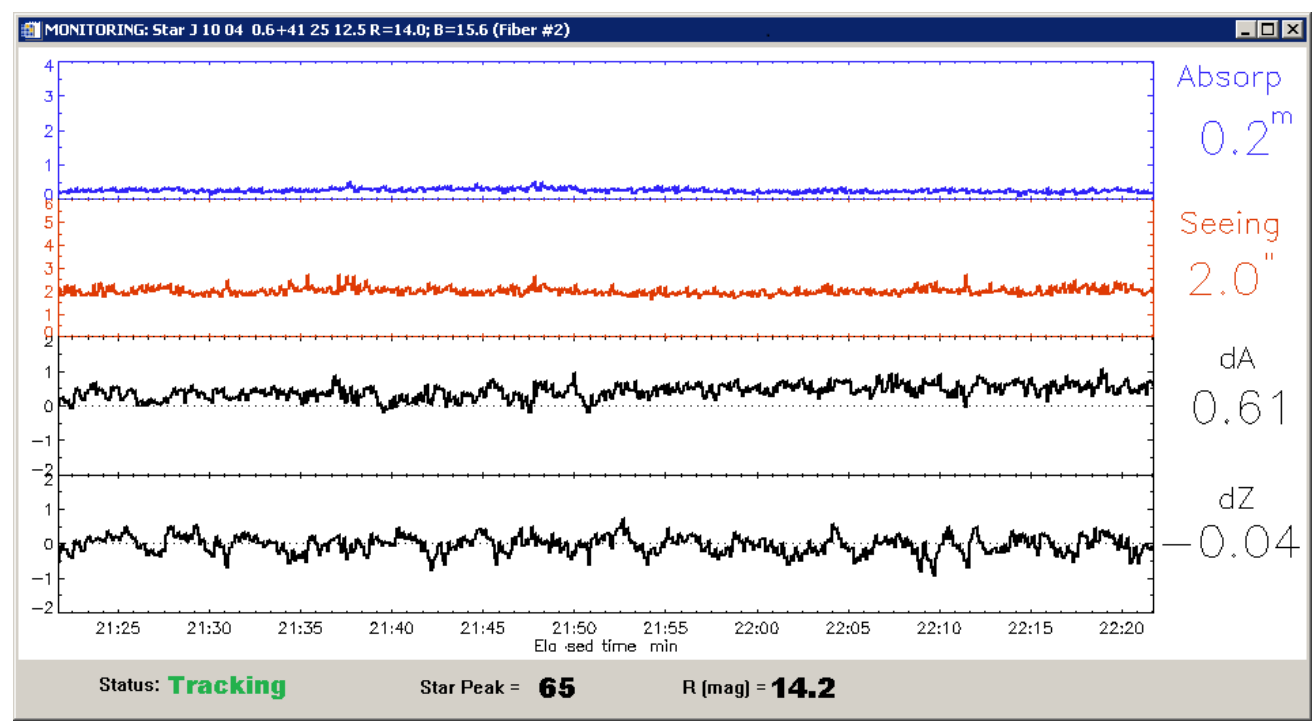

Figure 9. Interface of the monitoring window of the image of the guide star. The window title gives the coordinates of the star, USNO $2.0 R$ - and $B$-band magnitudes, and the number of the fiber bundle. The plots (from top to bottom): $R$-band absorption, seeing, offset of the centroid of the guide star along the azimuth and zenith distance (in arcsec). Right: the current parameter values. Bottom: the status line.

\subsection{Focusing}

The entrance end of each guiding fiber bundle can move along the optical axis within 0-9.7 mm to achieve focusing of the guide star (see Fig. 1). This makes it possible to correct the telescope focus during a long (more one hour) series of spectroscopic observations. Observational experience on the 6-m telescope shows that during that time variations of the focal length caused by thermal deformations of the telescope tube beams may exceed $0.1-0.2 \mathrm{~mm}$, which is quite significant in the case of good seeing $\left(\theta<2^{\prime \prime}\right)$. With the telescope and guiding microscopes focused before the beginning of the exposure series, the best focus position can then be controlled using the mechanism of focusing of guiding fields, and a compensating correction can be applied to adjust the focal distance of the telescope. The presence of two guiding fields allows the focus to be controlled by one of the stars while the other field is used for telescope tracking.

\section{CALIBRATION UNIT}

\subsection{General Description}

The calibration unit consists of an integrating sphere (Ulbricht sphere), calibration illuminator optics, and control system. The integrating sphere has two line-spectrum illuminators, a continuum- 
spectrum illuminator, and 32 ports for mounting illuminating LEDs. The line-spectrum source used is a $\mathrm{He}-\mathrm{Ne}-\mathrm{Ar}$-filled SGZ-3S gas-discharge radio lamp. The sources of continuum spectrum are an Osram Halopar-16 50W quartz tube and an array of 32 LEDs.

The optics of the calibration illuminator produces at the reducer input a converging beam with the focal ratio equivalent to the focal ratio of the telescope $(f / 4)$. The image of the uniformly illuminated area at the input of the integrating sphere is formed at the same place where the image of the telescope mirror is located (the output pupil) and hence the telecentrism condition is fulfilled. The projected diameter of the illuminator area coincides with the diameter of the pupil. In our case the telecentrism condition is fulfilled to within $0.2 \%$ or better. Such a solution makes it possible to perform correct wavelength calibration using line-spectrum lamp and calibrate the transmission variations of the system across the field in different modes of spectroscopic observations ("flatfield").

The output area of the integrating sphere is illuminated in three ways:

- NEON: by a He-Ne-Ar-filled lamp for wavelength calibration. It is used in the modes of slit and integral-field spectroscopy, and also in observations with the scanning Fabry-Perot interferometer [10]. Calibration is usually performed every time when the zenith distance of the telescope changes by more than $10-20^{\circ}$ with the allowance for instrument flexures.

- QUARTZ: by a halogen-filled quartz-tube continuum-spectrum lamp for creating "flatfield." Calibration is usually performed at the beginning and at the end of observations made in the scanning interferometer mode, and several times during the night in spectroscopic observations made at different zenith distances. A serious downside of such illuminator is the abrupt decrease of the lamp brightness in the blue spectral range, because the intensity maximum is at the wavelength of about $1200 \mathrm{~nm}$. As a result, at wavelengths $\lambda<500 \mathrm{~nm}$ the observed spectrum of the lamp is significantly contaminated by the scattered light from the longer-wavelength part of the spectrum. The use of equalizing filters slightly improves the situation.

- LEDS: by a system of light-emitting diodes that forms continuum spectrum for the "flatfield" with approximately uniform intensity distribution over a wide range of wavelengths. This allows performing uniformly precise flatfield illumination in different spectral ranges and reducing stray light in the blue region. Such a solution was first proposed for photometric calibration on small telescopes $[11,12]$ and it has so far not been used on large telescopes. For example, a system of 18 LEDs is planned to be used in the "white light" illuminator of EXPRES spectrograph [13].

Let us now consider in detail the latter illumination option provided by light-emitting diode system LEDS. 


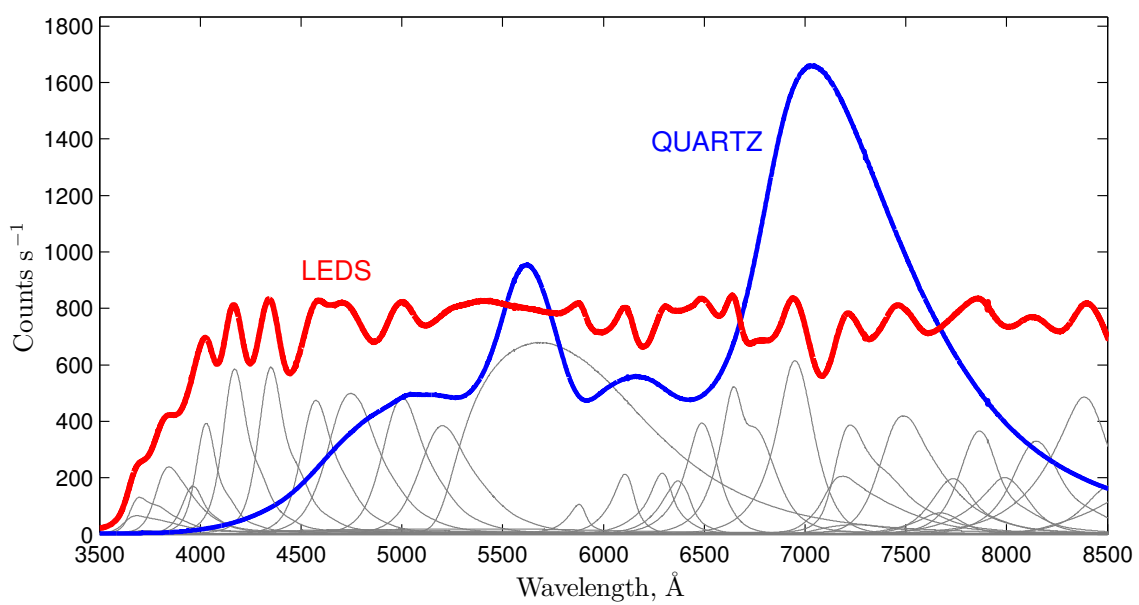

Figure 10. Spectral "flatfields" for VPHG940@600 grism obtained with QUARTZ quartz tube (with an equalizing filter) and an array of light-emitting diodes (LEDS). The numbers indicate the spectral curves of the corresponding LEDs. Spectra are normalized to 1-s exposure.

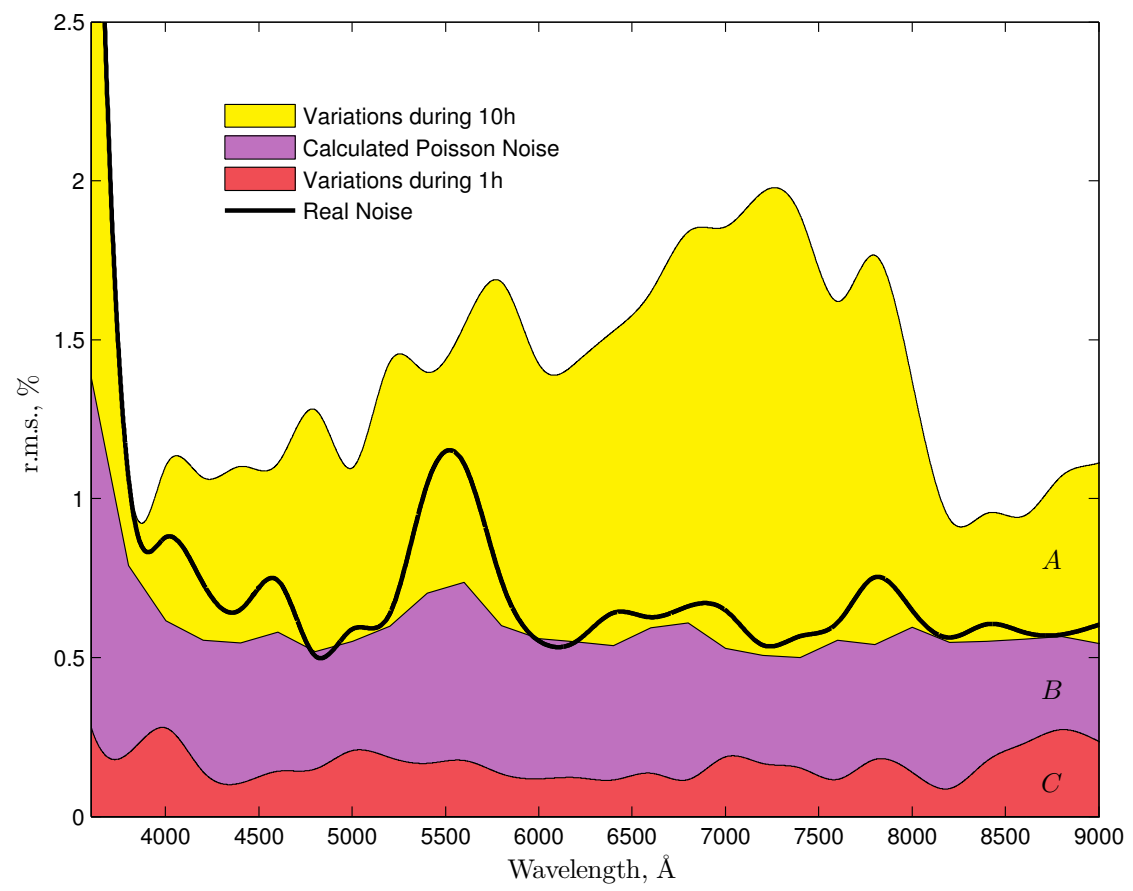

Figure 11. Wavelength dependence of relative variations of spectral "flatfield" with VPHG940@600 grism obtained with LED illumination.

\subsection{Light-Emitting Diode System}

Modern industry offers a wide range of various light-emitting diodes (LEDs) for the entire optical wavelength range. Each LED emits continuum spectrum in a narrow, about 10-20 nm wide 
wavelength interval. The brightness of individual elements in such a LED array can be selected so as to construct spectral "flatfield" for various grisms taking into account spectral sensitivity of the instrument mounted on the adapter.

We use pulse-width modulation to control the brightness of individual LEDs. To each LED a rectangular-wave signal is sent with different duty ratio, which varies discretely from 0 to 253 . Such realization is easy to implement and it ensures high stability of LED brightness. LEDs are controlled by the microprocessor mounted on the second board (see Section 2.4). The program started at the control computer in the IDL environment allows choosing either standard illumination configuration or setting the brightness of LEDs individually.

To illustrate LEDS technique, we show in Fig. 10 an example of the "flatfield" constructed for VPHG940@600 grism with the operating wavelength interval 3500-8500 A.

\subsection{Stability of LEDs}

The calibrating lamp consisting of a set of LEDs must ensure repeatability of the form of the spectrum in the temperature interval $\Delta T=-20 \ldots+40^{\circ} \mathrm{C}$. We estimate the stability of the luminous flux of the diode due to the change of external temperature $T$ from the following empirical relation adopted from [14]:

$$
F=F_{300} \exp \left(-\frac{T[\mathrm{~K}]-300}{T_{1}}\right)
$$

where $F_{300}$ is the LED flux at the close-to-room temperature and $T_{1}$ is the characteristic temperature for the given class of LEDs. The experimental $T_{1}$ values for blue, green, and red LEDs - 1600, 295, and $95 \mathrm{~K}$, respectively, are adopted from [14]. As is evident from equation (1), the flux of red LEDs is most sensitive to temperature variations and therefore the variations of the form of the spectrum should be the strongest in this wavelength range. Let us estimate its stability adopting the sensitivity of the flux of red LEDs as the worst case. Let us differentiate expression (1):

$$
d F / F_{300}=-\exp \left(-\frac{T-300}{T_{1}}\right) \frac{d T}{T_{1}}
$$

and compute the temperature coefficient of flux in the case of the change of the temperature by one degree.

To illustrate the effect, we list in the table the characteristic values of derivatives in the operating temperature range for red LEDs. 
Temperature coefficient for red LEDs

\begin{tabular}{c|c|c|c|c}
\hline$T,{ }^{\circ} \mathrm{C}$ & -20 & 0 & 27 & 40 \\
\hline$d F / F_{300}$ & -0.017 & -0.014 & -0.0105 & -0.0009 \\
\hline
\end{tabular}

When estimating the possible fluctuations of the spectrum of the lamp one must take into account the fact that during spectral exposure (5 to 60 minutes) the temperature at the primary focus of the telescope does not change by more than $1-2^{\circ} \mathrm{C}$.

To estimate the actual stability of LED illumination, two series of spectral "flatfields" were obtained in the laboratory with SCORPIO-2 using VPHG 940@600 grism: one series was acquired over 10 hours at 1-hour intervals, and another series was acquired over 1 hour at 1-minute intervals. Figure 11 shows the relative variations of the "flatfield" as a function of wavelength. Domain $A$ shows the scatter for the 10-hour experiment, which approximately simulates the situation for a single observing night. For comparison, we show domain $B$, which corresponds to computed Poisson noise. We calculated Poisson noise as $1 / \sqrt{N_{s} \times \text { gain }}$, where $N_{s}$ is the number of counts at the given wavelength and gain is the gain of the CCD. The thick line shows the actual noise in the spectrum, which agrees well with the theoretical level. Domain $C$ shows the scatter for the 1-hour experiment, which approximately simulates the situation for observations of a single object. One-hour variations $(C)$ can be seen to be lower than Poisson noise $(B)$. We determined the variations of both data series and the Poisson noise within $200 \AA$-wide bins.

Note that despite small variations of the ambient temperature over 10 hours (they remain within $5{ }^{\circ} \mathrm{C}$ limits), the amplitude of variations can be seen to gradually increase with wavelength from $1 \%$ to $2 \%$. Variations drop to $1 \%$ after $7500 \AA$ and this behavior reflects the contribution of the second order of VPHG 940@600 grism, and illumination in this wavelength range is also produced by blue LEDs.

Thus the "flatfield" produced using LEDS technique yields more uniform illumination compared to the "flatfield" produced using QUARTZ technique (Fig. 10). It would be reasonable to suggest that the hybrid QUARTZ+LEDS "flatfield", where LEDS corrects the drawbacks of QUARTZ technique in the blue part of the spectral range, should be the optimum solution. However, the LEDs and the quartz tube are located too close to each other, and the quartz tube heats up strongly when turned on, and therefore the LED properties change when both types of illumination are used simultaneously. This fact should be taken into account in observations and when designing similar systems. In the case of spectroscopic observations performed during the night we recommend using LEDS before QUARTZ (if both types of illumination are necessary), and separate their use in 
calibrations before and after observations.

The fact that LEDs are now widely used and relatively inexpensive suggests that such technique can be easily implemented on modern spectrographs.

\section{CONCLUSIONS}

The latest, fourth version of the adapter, which, like the previous two versions, was developed at the Laboratory of spectroscopy and photometry of extragalactic objects of the Special Astrophysical Observatory of the Russian Academy of Sciences, has been used for observations with SCORPIO-2 focal reducer [15] since 2012 .

Compared to the previous version of the adapter, which is still (summer 2017) used with SCORPIO, the new version has substantially reduced chromatic aberrations in guiding fields, and the spectral flatfield calibration is now formed by the combined emission of the LED array, which made it possible to increase the signal in the blue part of the spectrum multiple times.

Let us now point out the main features of the presented adapter:

1. To transfer the images of the guide stars to the guiding CCD two fiber bundles are used with possible individual focusing during observations.

2. The use of off-axis lens corrector made it possible to correct coma of the guide star images up to about $15^{\prime}$ from the optical axis.

3. The optics of the calibration illumination is made to fulfil the telecentrism condition.

4. LED system allows achieving relatively uniform spectral illumination of the CCD taking into account its response curve and the transmission curves of the grisms.

5. Photometry of the images of guide stars during observations is used to qualitatively control atmospheric transmittance and focusing of the telescope.

The guiding and calibration system described has been successfully tested for many years on the 6-m telescope of the Special Astrophysical Observatory of the Russian Academy of Sciences. 


\section{ACKNOWLEDGMENTS}

This work is supported by the Russian Science Foundation (grant no. 17-12-101335). We are grateful to E. A. Ivanov for developing the control program for the TV tuner.

1. B. Ioannisiani, Bull. Spec. Astrophys. Obs. 3, 3 (1971).

2. S. Milner and M. Ziebell, Messenger 8, 14 (1977).

3. D. McMullan and R. Powell, New Scientist 73, 715 (1977).

4. I. Karachentsev, Astronomy Letters 6, 3 (1980).

5. V. Afanasiev, V. Vlasyuk, S. Dodonov, and O. Sil'chenko, Preprint No. 54, SAO RAS (Spec. Astrophys. Obs., Nizhnij Arkhyz, 1990).

6. V. Afanasiev, S. Dodonov, and A. Moiseev, in Stellar Dynamics: From Classic to Modern, Ed. by L. P. Ossipkov and I. I. Nikiforov (Sobolev Astron. Inst., St.-Petersburg, 2001), p. 103.

7. V. Afanasiev, V. Vlasyuk, S. Dodonov, and S. Drabek, Report No. 242, SAO RAS (Spec. Astrophys. Obs., Nizhnij Arkhyz, 1995).

8. V. L. Afanasiev and A. V. Moiseev, Astronomy Letters 31, 194 (2005).

9. V. Afanasiev, E. Gazhur, S. Zhelenkov, and A. Moiseev, Bull. Spec. Astrophys. Obs. 58, 90 (2005).

10. A. V. Moiseev, Bull. Spec. Astrophys. Obs. 54, 74 (2002).

11. J. L. Marshall and D. L. DePoy, arXiv:astro-ph/0510233 (2005).

12. J. L. Marshall and D. L. DePoy, Publ. Astron. Soc. Pacific 125, 1277 (2013).

13. C. Jurgenson, D. Fischer, T. McCracken, et al., Proc. SPIE 9908, 99086T (2016).

14. E. F. Shubert, Light-Emitting Diodes (Cambridge Univ. Press, 2006).

15. V. L. Afanasiev and A. V. Moiseev, Baltic Astronomy 20, 363 (2011). 